\title{
Optimization Models and Solution Algorithms for Freight Routing Planning Problem in the Multi-Modal Transportation Networks: A Review of the State-of-the-Art
}

\author{
Yan Sun ${ }^{1,2, *}$, Maoxiang Lang ${ }^{1,2, *}$ and Danzhu Wang ${ }^{1,2}$ \\ ${ }^{1}$ School of Traffic and Transportation, Beijing Jiaotong University, Beijing 100044, P.R. China \\ ${ }^{2}$ MOE Key Laboratory for Urban Transportation Complex Systems Theory and Technology, Beijing Jiaotong \\ University, Beijing 100044, P.R. China
}

\begin{abstract}
With the remarkable development of international trade, global commodity circulation has grown significantly. To accomplish commodity circulation among various regions and countries, multi-modal transportation scheme has been widely adopted by a large number of companies. Meanwhile, according to the relevant statistics, the international logistics costs reach up to approximate $30-50 \%$ of the total production cost of the companies ${ }^{1}$. Lowering the transportation costs has become one of the most important sources for a company to raise profits and maintain competitiveness in the global market. Thus, how to optimize freight routes selection to move commodities through the multi-modal transportation network has gained great concern of both the decision makers of the companies and the multi-modal transport operators. In this study, we present a systematical review on the multi-modal transportation freight routing planning problem from the aspects of model formulation and algorithm design. Following contents are covered in this review: (1) distinguishing the formulation characteristics of various optimization models; (2) identifying the optimization models in recent studies according to the formulation characteristics; and (3) discussing the solution approaches that are developed to solve the optimization models, especially the heuristic algorithms.
\end{abstract}

Keywords: Freight routing planning problem, multi-modal transportation, optimization models, formulation characteristics, heuristic algorithms.

\section{INTRODUCTION}

Routing planning is a kind of combinatorial optimization problem that is related to the optimal utilization of the resources in a system. By selecting optimal routes to distribute the flow of the objects (e.g. commodities, products, data, signals, etc.) in systems, the performance of the systems can be improved efficiently from many viewpoints (economy, stability, timeliness, etc.). Consequently, routing planning optimization has been paid great attention not only in transportation, but also in many other fields such as telecommunication [1-3], manufacturing systems [4-6] and Internet service networks [7-9].

The freight routing planning aims at assigning optimal routes to move commodities from their origins to the respective destinations through the transportation networks. In the freight transportation network design, planning has three levels, including strategic planning, tactical planning and operational planning [10], and the freight routing

*Address correspondence to these authors at the Beijing Jiaotong University, No. 3 Shangyuancun, Haidian District, Beijing, P. R. China 100044; Tel: 86-10-51684152; E-mails: sunyanbjtu@163.com; mxlang@bjtu.edu.cn

${ }^{1}$ Data Source: H. Min, "International intermodal choices via chance-constrained goal programming”, Transportation Research Part A: General, vol. 25, pp. 351-362, November 1991. planning belongs to the third level. Although it is a short term decision making in the transportation network design [11], freight routing planning is oriented directly on satisfying the customers' demand, and its performance determines the competitiveness of a transportation carrier or a third party logistics company in the freight market.

For multi-modal transportation, freight routing planning refers to the combinations of various transportation modes (rail, road, water and air) to generate origin-to-destination routes to move commodities through the multi-modal transportation networks. The truck-rail and rail-ocean combinations are the prevalent manners in the practical multi-modal transportation [12].

Respective advantages of the transportation modes above are integrated in the freight routing planning when adopting multi-modal transportation, and the employment of multimodal transportation freight routes has been empirically proved to be a more cost-effective [13] and eco-friendly (less carbon emissions) [14] way than the uni-modal transportation. However, compared with the uni-modal transportation network, the operating distinction among the transportation modes makes the multi-modal transportation freight routing planning more complicated.

The operation in the multi-modal transportation has two main categories: scheduled service pattern (e.g. railway 
freight transportation) and time-flexible service pattern (e.g. road freight pick-up and delivery services) [15]. The scheduled service means the following contents of a transportation mode are all prescribed manually: terminal sequence on the operating itinerary, arrival times at the terminals, departure times from the terminals, loading start times and loading cutoff times at the terminals, carrying capacities at the terminals and the service frequency. All the time related parameters of the commodities (arrival time, departure time, inventory time, transshipping time, etc.) depend on the connecting manner of the two kinds of transportation modes (scheduled one to scheduled one, scheduled one to time-flexible one, time-flexible one to timeflexible one, time-flexible one to scheduled one).

In a word, the multi-modal transportation freight routing planning is a comprehensive optimization problem that takes the customers' demands, service patterns of various transportation modes and network resources as well as the transportation cases into consideration.

In this study, we present a systematical review on the freight routing planning problem in the multi-modal transportation network. In Section 2, we focus on optimization models on the multi-modal transportation freight routing problem, compare different formulation characteristics and identify the optimization models in the current studies according to the formulation characteristics. In Section 3, we discuss the solution approaches that are developed to solve the optimization models, especially the heuristic algorithms. Finally, the conclusions of this study are drawn in Section 4.

\section{MULTI-MODAL TRANSPORTATION FREIGHT ROUTING PLANNING MODELS}

Optimization models are formulated to describe the multi-modal transportation freight routing planning problem mathematically. By inputting the practical transportation data into the optimization models and then solving them by exact solution methods (e.g. column generation method [16] and branch-and-bound method [17]) or approximate solution methods (e.g. Genetic algorithm [18] and Tabu search algorithm [19]), optimal solutions can be attained and can provide decision makers with quantitative decision support. In the last decades, many studies devoted themselves to optimizing the multi-modal transportation freight planning problem. The growth of research interest has motivated the emergence of a new applied transportation research field [20, 21].

Nearly all the optimization models for the multi-modal transportation freight routing planning problem are established on the mixed integer linear/nonlinear programming. In the model formulation, models with different formulation characteristics are suitable for problems with different requests and situations. Thus it is necessary to distinguish the formulation characteristics shown in Fig. (1).

\subsection{Optimization Object: Single Commodity vs. Multiple Commodities}

In the multi-modal transportation network, single commodity corresponds to an OD (origin terminal-todestination terminal) pair. Models whose optimization object is one commodity concentrate on the multi-modal transportation freight routing planning for a specific customer with transportation demand, e.g. Barnhart and Ratliff [22], Xiong and Wang [18] and Lei et al. [23]. In these studies, an optimal route is needed to move the commodity from its origin to its destination through the multi-modal transportation network. Under this circumstance, transportation service for this commodity will reach its optimum.

Actually, in the entire multi-modal transportation network within a geographic space, more than one commodity has to be moved through the network. Different

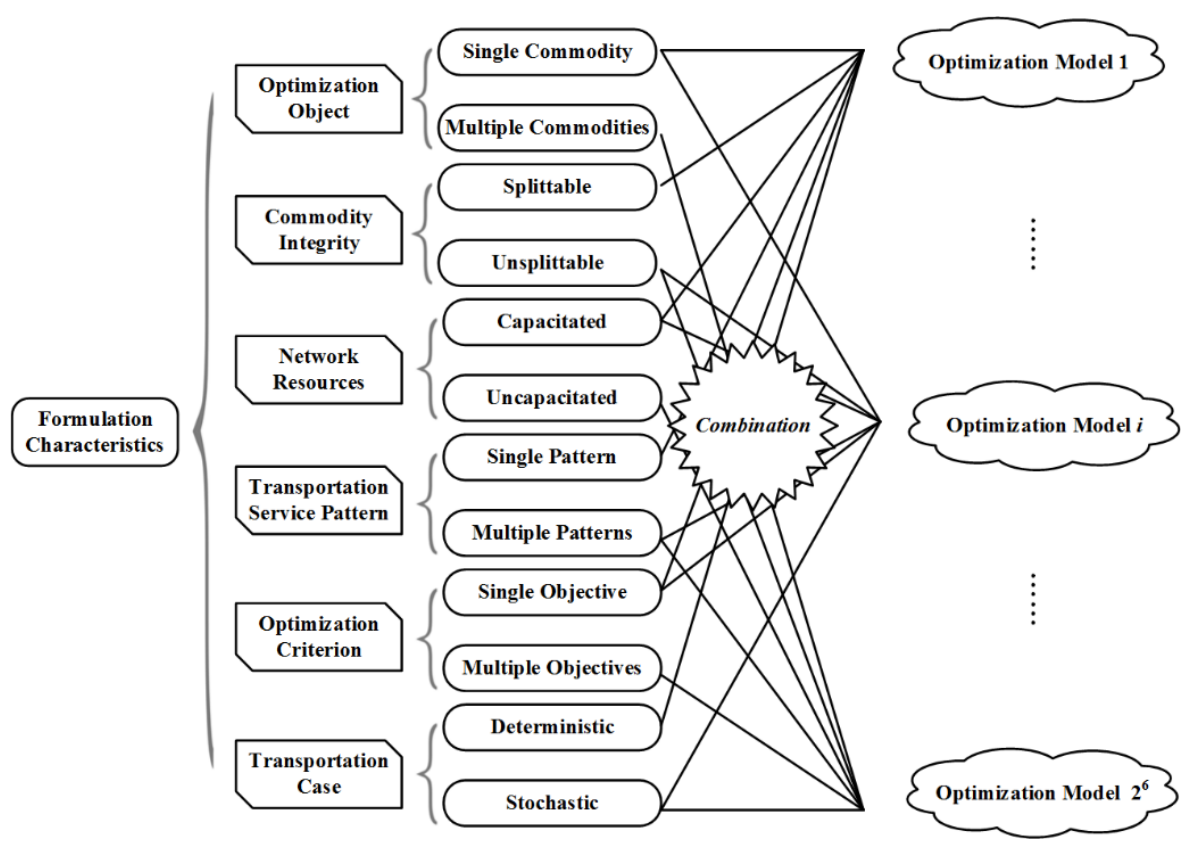

Fig. (1). Formulation characteristics of the optimization models. 
commodities have different categories, freight volumes, release times, transit periods, and correspond with different OD pairs. Optimum for the transportation of one commodity does not equal to the optimum for the overall service performance of the multi-modal transportation network. For the purpose of network/system service performance optimum, many studies expanded the optimization object to the multiple commodities (specifically, origin-destinationcommodity combinations [24]) in the multi-modal transportation network, e.g. Bookbinder and Fox [25], Chang [12] and Holguín-Veras and Pati [26]. In these studies, the problem is defined as the multi-commodity multi-modal transportation freight routing planning problem. Under this circumstance, the entire multi-modal transportation network service performance will reach its optimum.

\subsection{Commodity Integrity: Splittable vs. Unsplittable}

In many studies by Zhang et al. [27], Liu et al. [28], Li et al. [29], etc., each commodity is not allowed to be split into several sub commodities, and its transportation should follow one route through the multi-modal transportation network (see Fig. 2-a) exactly [11]. In the relevant models, an $0-1$ variable $x_{i j m}^{k}$ is defined that if the directed arc $(i, j)$ is used to move the commodity $\mathrm{k}$ by transportation mode $\mathrm{m}$, $x_{i j m}^{k}=1$, otherwise $x_{i j m}^{k}=0$. And $x_{i j m}^{k}$ should satisfy the following constraint (Eq.1) so as to ensure the commodity is unsplittable.

$\sum_{\mathrm{m}} \mathrm{x}_{\mathrm{ijm}}^{\mathrm{k}} \leq 1 \forall \mathrm{k} \forall(\mathrm{i}, \mathrm{j})$

While in other studies by Chang [12], Qu et al. [30], etc., each commodity is considered to be splittable, and one commodity can follow several routes from its origin to its destination (see Fig. 2-b). By dropping the constraint of the 0-1 variable above, the unsplittabe multi-modal transportation freight routing planning can be converted into the splittable one [31].

\subsection{Network Resources: Uncapacitated Network vs. Capacitated Network}

Network resources are the facilities in the multi-modal transportation network, which can be classified into two categories: fixed facilities (e.g. yards, warehouses and transportation tracks) and movable facilities (e.g. rail wagons, locomotives, vessels, aircrafts and trucks). Network resources construct the material basis of multi-modal transportation freight routing planning. In other words, the multi-modal transportation freight routing planning addresses the utilization of the multi-modal transportation network resources to organize the freight transportation according to the customers' demands.

In most cases, the resources available for the freight routing planning in a specific multi-modal transportation network are limited, due to the restriction of the facilities in number and their limited workload as well as other transportation services that occupy parts of the multi-modal transportation network resources. Additionally, other factors, e.g. transportation congestion, also make it hard to assign routes to move commodities through the multi-modal transportation network at will. In the model formulation, capacity is used to measure the restriction of the multi-modal transportation network resources.

Many studies by Balakrishnan et al. [32], Xiong and Wang [18], Wang and Han [33], etc., do not take the capacity constraints into consideration, and the multi-modal transportation network is hence uncapacitated. It should be noted that the freight routes planned by these models may be infeasible in the practice according to the analysis above. As highlighted by Holmberg and Yuan [34] and Chang [12] and Cho et al. [35], capacitated multi-modal transportation freight routing planning, , can avoid generating freight routes that exceed the resource restriction of the multi-modal transportation network, and meets the practical transportation

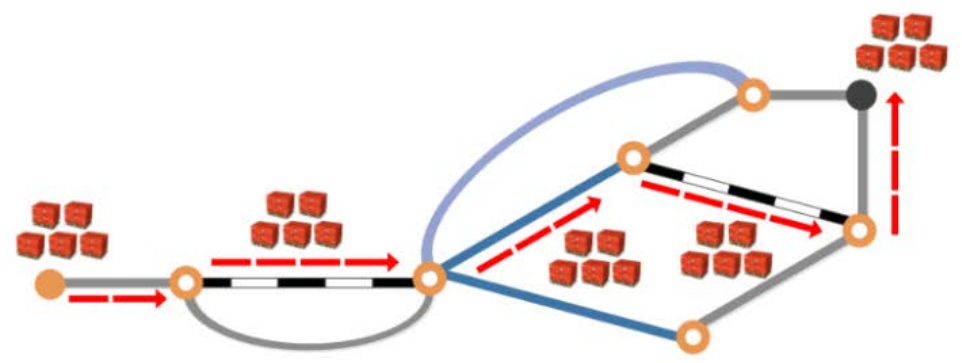

(a) unsplittable commodity flow

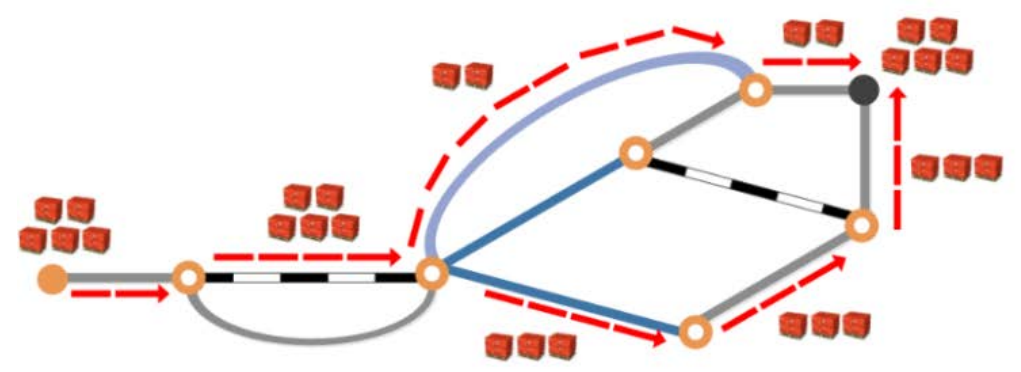

(b) splittable commodity flow

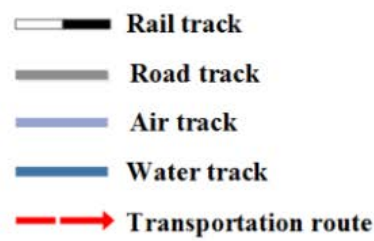

Origin terminal

Transfer terminal

Destination terminal

Fig. (2). Unsplittable and splittable commodity flow. 
demands better than the uncapacitated freight routing planning. In the capacitated freight routing planning, inventory capacity of the terminals and the carrying capacity of the vehicles are usually included in the constraints of the models.

In the capacitated freight routing planning, when the optimization object is multiple commodities, different commodities occupy the terminals and vehicles in different time intervals. For commodities whose time intervals have intersections, the summation of their volume should not exceed the two kinds of capacities above.

\subsection{Transportation Service Pattern: Single Pattern vs. Multiple Patterns}

Many studies by Sun et al. [36], Xiong and Wang [18] and Lei et al. [23] etc., considered all the transportation modes adopted the time-flexible service pattern, and the main procedure of the commodities at the terminals can be simply summarized as "arrival $\rightarrow$ transshipment $\rightarrow$ departure". In fact, apart from the time-flexible service pattern, there also exists scheduled service pattern in the multi-modal transportation network. Freight routing planning solves the connecting issues of the multiple service patterns. So many studies considered multiple service patterns in the optimization models comprehensively, as highlighted by Moccia et al. [15, 37], Liu et al. [28] and Ayar and Yaman [38] etc. When adopting the scheduled services, the moving of the commodities should exactly follow the schedules of the services.

Assume the loading start time and loading cutoff time of the scheduled service $\mathrm{s}$ at terminal $\mathrm{i}$ are $\mathrm{st}_{\mathrm{s}}^{\mathrm{i}}$ and $\mathrm{ct}_{\mathrm{s}}^{\mathrm{i}}$, respectively, the arrival time of commodity $\mathrm{k}$ at this terminal is $\mathrm{a}_{\mathrm{i}}^{\mathrm{k}}$, and time consumed to load commodity $\mathrm{k}$ on services $\mathrm{s}$ is $t_{i}^{\mathrm{ks}}$.

If the commodity is able to use this service, there should be $a_{i}^{k}+t_{i}^{k s} \leq s t_{s}^{i}$. When $a_{i}^{k}<s t_{s}^{i}$, commodity $k$ waits at terminal i until $s t_{s}^{i}$, then it starts to be loaded on service $s$. Under this circumstance, $\mathrm{t}_{\mathrm{i}}^{\mathrm{ks}}$ should satisfy $\mathrm{t}_{\mathrm{i}}^{\mathrm{ks}} \leq \mathrm{ct}_{\mathrm{s}}^{\mathrm{i}}-\mathrm{st}_{\mathrm{s}}^{\mathrm{i}}$. After loading on service $\mathrm{s}$, commodity $\mathrm{k}$ finally departs from terminal $i$ at the scheduled departure time of service $s$ at this terminal.

\subsection{Optimization Criterion: Single Objective vs. Multiple Objectives}

Satisfying the transportation demand at minimal costs is the most important criterion for the multi-modal transportation freight routing planning. Costs optimum has always been the optimization objective of the models, especially in the single objective optimizations, as delineated by Song and Chen [39], Li et al. [40], etc., and the aim of these single objective optimizations is to select the most cost-efficient routes to move commodities through the multimodal transportation network.

Besides the costs optimum, improving the transportation efficiency and abating environmental pollution have also received great importance. To address the two requests, lowering the transportation time and reducing the carbon emissions are also set as the optimization objectives in the routing planning. Thus many studies established multiobjective optimization models to identify rational routes that can make a tradeoff between costs optimum and transportation efficiency optimum (e.g. Chang [12], Xiong and Wang [18] and Lei et al. [23]) or between costs optimum and carbon emissions optimum (e.g. Sun and Chen [41]). Existing methods to deal with the multiple objective optimizations of the multi-modal transportation freight routing planning include: weighted sum method and Pareto optimality.

The first method distributes different weights to the objectives and then combines them linearly [42]. The multiobjective optimization is converted into a single objective one. Chang [12] and Lei et al. [23] adopted this method in their studies.

The second one aims to gain the Pareto frontier (evendistributed Pareto solutions) of the multi-objective optimization models [42]. Xiong and Wang [18] adopted this method in their study. The widely applied approach to gain the Pareto frontier of the multi-objective optimizations is the normalized normal constraint method. The details of this method are introduced in Messac et al.'s study [43].

Moreover, other methods, e.g. lexicographic goal programming approach [44], can be also adopted to solve the multi-objective optimizations according to the practical situation.

\subsection{Transportation Case: Deterministic Case vs. Stochastic Case}

Almost all the studies focus on the model formulation that is subject to deterministic cases. In the relevant studies, following parameters are all set as fixed values: Transportation demand; travel time (e.g. Chang [12] and Lei et al. [23]) or travel speed (e.g. Xiong and Wang [18]) on tracks; transshipping time at terminals. In many studies, transshipping time is only determined by the connecting manners of the two transportation modes (e.g. Jiang and $\mathrm{Lu}$ [45], Wang and Han [33] and Lei et al. [23]).

However, on the one hand, many studies have pointed out that the transportation demands show high uncertainty over space and time [46], and satisfying fluctuating transportation demand is of great challenge for decision makers to plan multi-modal transportation freight routes in mid-and-long term [47]; on the other hand, because of the influence of environmental factors, e.g. weather variation, traffic congestion, emergency, etc., the travel times or travel speeds of the transportation modes on tracks and operating time at terminals are hard to remain stable constantly and present obvious uncertainty. All these factors mentioned above also increase the delay probability of scheduled transportation modes at terminals. Together with the difference of the resource distribution to different terminals, transshipping time at different terminals is impossible to remain identical and presents uncertainty as well [48].

Therefore, uncertainties are widespread phenomena in the multi-modal transportation network, and the estimation 
of the uncertainties can help identify the real-world multimodal transportation freight routing planning [47], while there are only a few studies that relate to the model formulation based on stochastic cases. Wang et al. [48] used the Normal distribution to describe the uncertainty of the operating time of the containers at terminals. Chen and Sun [41] considered the travel time of the transportation modes on tracks and operating time at terminals to be stochastic and built a chance-constrained mixed integer programming model. In this study, Monte Carlo Method and Normal distribution hypothesis based deterministic equivalent form are used to solve the stochastic problem.

\subsection{Formulation Characteristics of Optimization Models in Recent Studies}

According to the analysis above, the formulation characteristics of the optimization models in recent studies are identified in Table $\mathbf{1}$.

\section{SOLUTION APPROACHES}

The multi-modal transportation freight routing problem is known as a kind of NP hard problems [12, 18], and this characteristic has also been verified in Ayar and Yaman's study [38], i.e. the optimal solutions of the large-scale or real-world freight routing planning problem are hard to be attained by the single exact solution methods [53], while the heuristic algorithms proved good feasibility in solving the optimization models, e.g. Genetic algorithm, PSO algorithm and ACO algorithm, etc. [18, 29, 40, 41, 52].

The heuristic algorithms as well as their optimizations and variations are various, and the mechanics of different heuristic algorithms differs from each other, including the representations of the solutions, evaluation manners of the solutions, updating strategies of the solutions and the termination criteria. However, all the heuristic algorithms share a basic flowchart shown in Fig. (3).

Among the various heuristic algorithms, Genetic algorithm is the most classic and popular one. A good understanding of the Genetic algorithm can help us capture the essence of the heuristics.

Genetic algorithm was proposed by Holland in 1975. It is an optimization technique based on the mechanics of natural genetics. In the genetic algorithm, solutions of the optimization problem are represented by the encoding of chromosomes (gene sequences).In the iteration process, the solutions are modified by the selection, the crossover between different chromosomes and the mutation of the chromosomes. The fitness function is used to evaluate the quality of the solutions. The chromosome whose corresponding solution has larger fitness value has higher probability to be selected to enter the next iteration $[54,55]$. The basic components of the Genetic algorithm are stated as follows.

\section{(1) Representation of the solutions}

Encoding of the chromosomes/gene sequences.

(2) Evaluation of the solutions
The fitness function is used to evaluate the quality of a solution. Generally, the quality of a solution is proportional to its fitness value. Therefore, for the minimization problem $\min z$, the fitness function is usually set as $f=1 / z$.

(3) Updating strategy

a. Selection

The common selection strategy is proportional selection. In the $t^{\text {th }}$ iteration, let $F_{i}^{t}$ and pop denote the fitness value of the $i^{\text {th }}$ chromosome (gene sequence) and the size of the population (chromosome set), respectively. The selection probability of this individual can be calculated by Eq. 2 .

$\mathrm{p}_{\mathrm{i}}^{\mathrm{t}}=\frac{\mathrm{F}_{\mathrm{i}}^{\mathrm{t}}}{\sum_{\mathrm{i}=1}^{\text {pop }} \mathrm{F}_{\mathrm{i}}^{\mathrm{t}}} \forall \mathrm{i}=1,2, \ldots$, pop

Then we can use Roulette Wheel to perform the selection operation. First we calculate the cumulative probability of the $\mathrm{i}^{\text {th }}$ chromosome by Eq. 3 .

$\mathrm{pp}_{\mathrm{i}}^{\mathrm{t}}=\left\{\begin{array}{c}0 \quad \mathrm{i}=0 \\ \sum_{\mathrm{j}=1}^{\mathrm{i}} \mathrm{p}_{\mathrm{j}}^{\mathrm{t}} \quad \forall \mathrm{i}=1,2, \ldots, \text { pop }\end{array} \forall \mathrm{i}=0,1, \ldots\right.$, pop

Then we generate a random number $u_{i}^{t} \in(0,1)$. If $p p_{i}^{t}>u_{i}^{t}>p p_{i-1}^{t}$, the $i^{\text {th }}$ chromosome will be maintained to the next iteration.

b. Crossover and mutation

The crossover between two different chromosomes and mutation of a certain chromosome can enhance the variety of the solutions of the problem. They appear under a small probability after the selection operation and their values are both set manually in the Genetic algorithm.

(4) Termination criterion

Genetic algorithm terminates after evolving a certain number of generations (iterations).

Above all, the flowchart of the Genetic algorithm is shown in Fig. (4) [56].

For the heuristic algorithms themselves, their performances are determined by the updating strategies in the updating step. Generally, good performance of a heuristic algorithm refers to: (1) good global searching capacity (exploration capacity) in the initial iteration stage of the heuristic algorithm that can avoid the local optimum; and (2) good local searching capacity (exploitation capacity) in the final stage that can accelerate the convergence to the global optimum [57]. To improve the performance of a specific heuristic algorithms, two kinds of methods can be utilized in its updating step, including coefficient modification method and combinatorial optimization methods.

The first method is to modify the values of the coefficients in the updating formulas dynamically during the iteration process to improve the updating step. The most typical heuristic algorithm using this method is PSO algorithm whose updating process can be seen in Sun et al.'s study [58]. In its iteration process, time linear decreasing inertia weight algorithm and time varying acceleration coefficient algorithm are both adopted to modify the inertia weight and acceleration coefficients in the particle velocity updating formula, respectively. By using the two coefficient modification algorithms, the inertia weight and local acceleration coefficient will decrease iteratively, while the 
Table 1. Formulation characteristics of the optimization models in recent studies.

\begin{tabular}{|c|c|c|}
\hline Author(s) & Year & Formulation Characteristics \\
\hline $\begin{array}{l}\text { Holmberg and Yuan } \\
\text { [34] }\end{array}$ & 2000 & $\begin{array}{c}\text { multiple commodities / splittable commodity / capacitated network / single service pattern / single objective } \\
\text { optimization / deterministic case }\end{array}$ \\
\hline $\begin{array}{l}\text { Wang and Wang } \\
\text { [49] }\end{array}$ & 2005 & $\begin{array}{c}\text { single commodity / unsplittable commodity / capacitated network / single pattern service / single objective } \\
\text { optimization / deterministic case }\end{array}$ \\
\hline Wei et al. [50] & 2006 & $\begin{array}{c}\text { single commodity / unsplittable commodity / uncapacitated network / single service pattern/ single objective } \\
\text { optimization / deterministic case }\end{array}$ \\
\hline Zhang et al. [27] & 2006 & $\begin{array}{c}\text { single commodity / unsplittable commodity / uncapacitated network / single service pattern / single objective } \\
\text { optimization / deterministic case }\end{array}$ \\
\hline Chang [12] & 2008 & $\begin{array}{l}\text { multiple commodities / splittable commodity / capacitated network / multiple service patterns / multi-objective } \\
\text { optimization / deterministic case }\end{array}$ \\
\hline Sun et al. [36] & 2008 & $\begin{array}{l}\text { single commodity / unsplittable commodity / uncapacitated network / single service pattern / single objective } \\
\text { optimization / deterministic case }\end{array}$ \\
\hline Moccia et al. [37] & 2008 & $\begin{array}{l}\text { multiple commodities / splittable commodity / capacitated network / multiple service patterns / single objective } \\
\text { optimization / deterministic case }\end{array}$ \\
\hline Jiang and Lu [45] & 2008 & $\begin{array}{c}\text { single commodity / unsplittable commodity / uncapacitated network / single service pattern / single objective } \\
\text { optimization / deterministic case }\end{array}$ \\
\hline Cai et al. [51] & 2010 & $\begin{array}{l}\text { single commodity / unsplittable commodity / uncapacitated network / single service pattern / single objective } \\
\text { optimization / deterministic case }\end{array}$ \\
\hline Wang and Han [33] & 2010 & $\begin{array}{l}\text { single commodity / unsplittable commodity / uncapacitated network / single service pattern / single objective } \\
\text { optimization / deterministic case }\end{array}$ \\
\hline Kang et al. [52] & 2010 & $\begin{array}{l}\text { single commodity / unsplittable commodity / capacitated network / single service pattern / multi-objective } \\
\text { optimization / deterministic case }\end{array}$ \\
\hline Liu et al. [28] & 2011 & $\begin{array}{l}\text { single commodity / unsplittable commodity / capacitated network / multiple service patterns / single objective } \\
\text { optimization / deterministic case }\end{array}$ \\
\hline Moccia et al. [15] & 2011 & $\begin{array}{l}\text { multiple commodities / splittable commodity / capacitated network / multiple service patterns / single objective } \\
\text { optimization / deterministic case }\end{array}$ \\
\hline Li et al. [40] & 2011 & $\begin{array}{c}\text { single commodity / unsplittable commodity / uncapacitated network / single service pattern / single objective } \\
\text { optimization / deterministic case }\end{array}$ \\
\hline Wang et al. [52] & 2011 & $\begin{array}{l}\text { single commodity / unsplittable commodity / capacitated network / single service pattern / single objective } \\
\text { optimization / deterministic case }\end{array}$ \\
\hline Wang et al. [48] & 2011 & $\begin{array}{l}\text { single commodity / unsplittable commodity / uncapacitated network / single service pattern / single objective } \\
\text { optimization / stochastic case }\end{array}$ \\
\hline $\begin{array}{l}\text { Ayar and Yaman } \\
\text { [38] }\end{array}$ & 2012 & $\begin{array}{l}\text { multiple commodities / unsplittable commodity / capacitated network / multiple service patterns / single objective } \\
\text { optimization / deterministic case }\end{array}$ \\
\hline Li et al. [29] & 2012 & $\begin{array}{l}\text { single commodity / unsplittable commodity / uncapacitated network / single service pattern / single objective } \\
\text { optimization / deterministic case }\end{array}$ \\
\hline Sun and Chen [41] & 2013 & $\begin{array}{l}\text { single commodity / unsplittable commodity / uncapacitated netowrk / single service pattern / multi-objective } \\
\text { optimization / stochastic case }\end{array}$ \\
\hline Lei et al. [23] & 2014 & $\begin{array}{l}\text { single commodity / unsplittable commodity / capacitated network / single service pattern / multi-objective } \\
\text { optimization / deterministic case }\end{array}$ \\
\hline $\begin{array}{l}\text { Xiong and Wang } \\
\text { [18] }\end{array}$ & 2014 & $\begin{array}{l}\text { single commodity / unsplittable commodity / uncapacitated network / single service pattern / multi-objective } \\
\text { optimization / deterministic case }\end{array}$ \\
\hline Qu et al. [30] & 2014 & $\begin{array}{c}\text { multiple commodities / splittable commodity / capacitated network / single service pattern / single objective } \\
\text { optimization / deterministic case }\end{array}$ \\
\hline
\end{tabular}

global acceleration coefficient will increase iteratively, which can avoid the particles into local optimal positions in the initial stage of the iteration process and accelerate their convergence to the global optimal position in the final stage. 
The second method is to combine different heuristic algorithms based on their respective advantages in searching capacities. The most common combinatorial optimization method is the Tabu-Genetic combinatorial algorithm. This

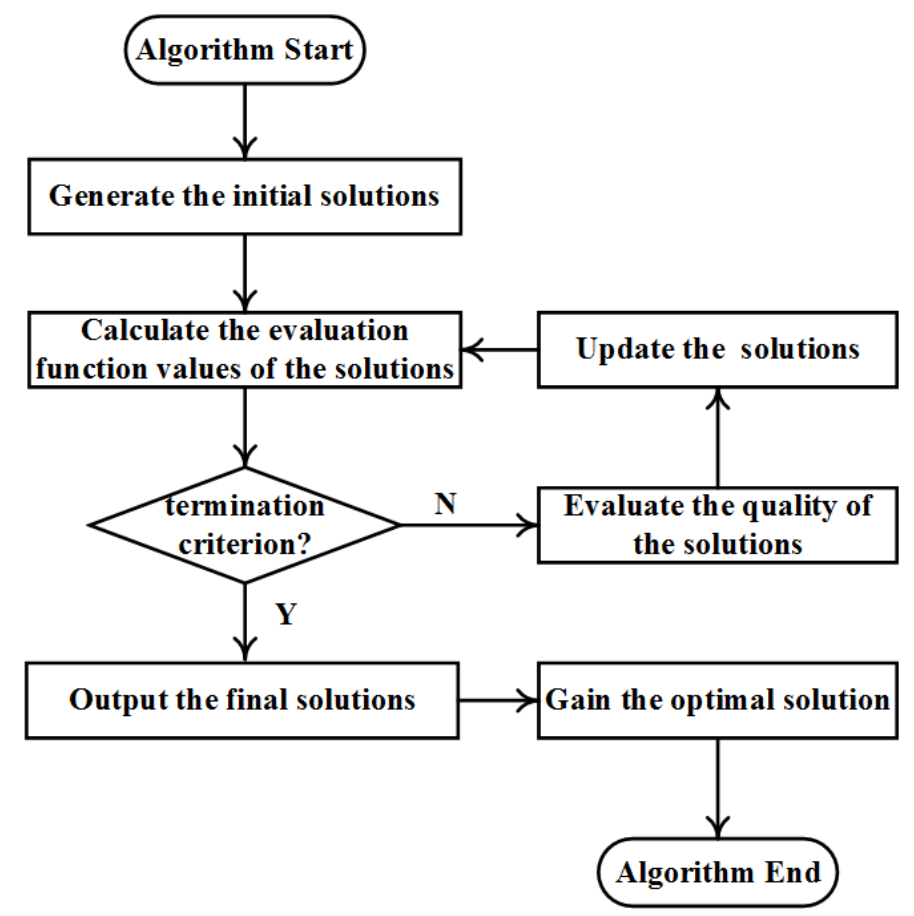

Fig. (3). Common flowchart of the heuristic algorithms.

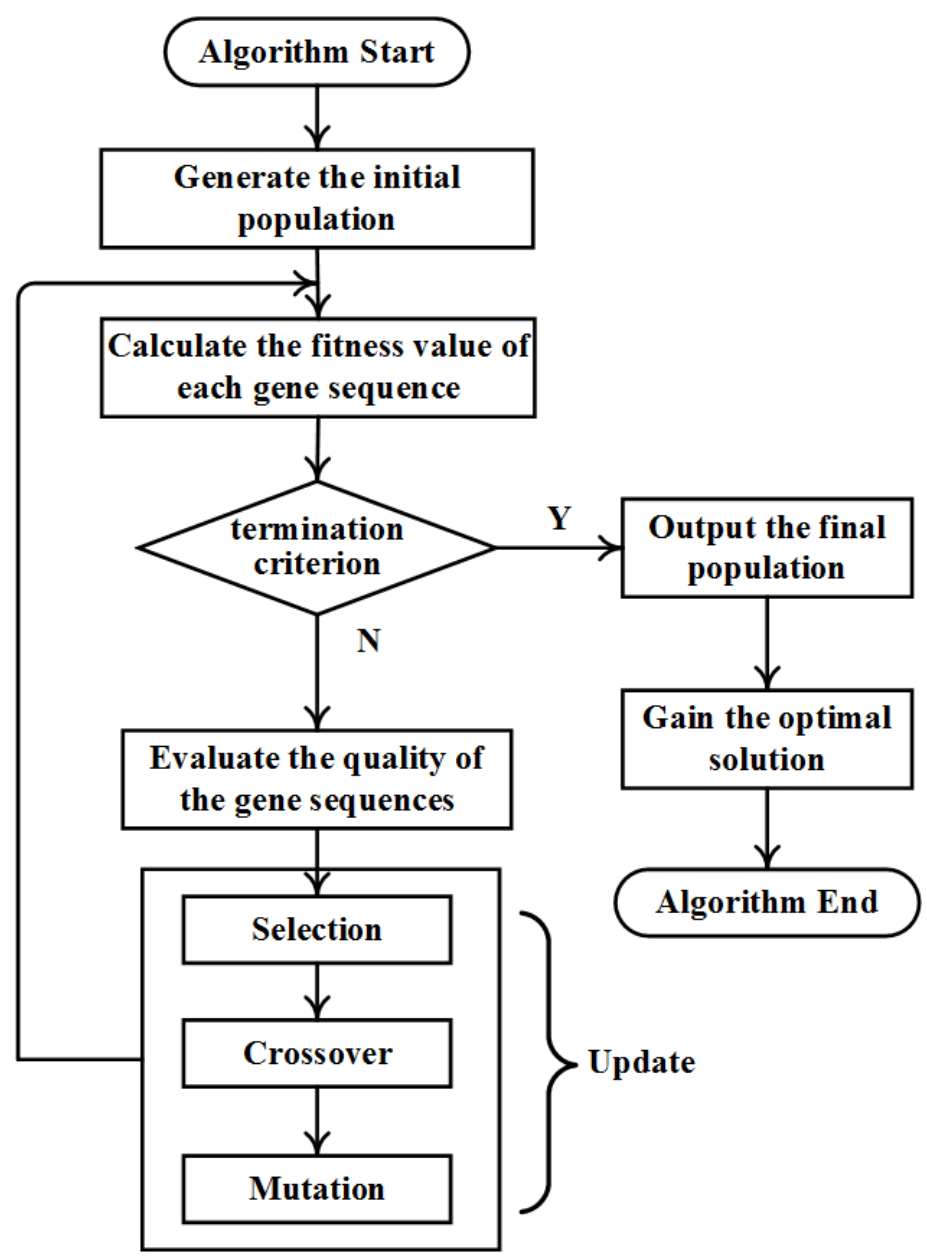

Fig. (4). Flowchart of the Genetic algorithm. 
algorithm combines the good capacities of Genetic algorithm in the global searching and Tabu search algorithm in the local searching, which can greatly improve the quality of the solutions when compared with the single Genetic algorithm or Tabu search algorithm. In the Tabu-Genetic combinatorial algorithm, the Genetic algorithm is set as the main algorithm, and Tabu search algorithm or only the Tabu list thought is incorporated into the updating strategy of the Genetic algorithm [57]. For more details of the Tabu-Genetic combinatorial algorithm, we can refer to Glover et al.'s study [59] or other related studies.

It should be noted that when using the heuristic algorithms alone to solve the problem optimally, it is hard to guarantee the solution optimality and efficiency of the heuristic algorithms themselves. To avoid the inherent weakness of the heuristic algorithms, it is better to first adopt small-scale cases to compare the solution optimality of the heuristic algorithms with the exact solution methods. The exact solution methods can be easily performed by the mathematical programming software, e.g. Lingo, CPLEX and GAMS. The nonlinear optimization models must be linearized before using the mathematical programming software. Then large-scale or real world cases can be adopted to compare the solution efficiency of the heuristic algorithms with the exact solution methods. The two-stage technique can demonstrate both the solution optimality and computational efficiency of the heuristic algorithms in solving the multi-modal transportation freight routing planning problem.

\section{CONCLUSION}

In this study, we present a systematical review on the freight routing planning problem in the multi-modal transportation network from the viewpoints of the model formulation and solving approaches. In this study, the formulation characteristics are distinguished and classified into six aspects, and the optimization models in the recent studies are identified based on their respective formulation characteristics. Furthermore, the solution approaches developed to solve the optimization models are discussed, especially the heuristic algorithms.

As we can see from the progress of current studies, almost all of them simplified the multi-modal transportation freight routing planning problem excessively. Some necessary factors, such as multi-commodity flow, multiple services (especially the scheduled services), and stochastic case, are ignored. Still there are a few valuable researches, such as Chang's [12], Moccia et al.'s [15, 37] and Ayar and Yaman's [38]. In our opinion, future researches should focus on the stochastic multi-commodity multi-modal freight routing problem that considers multi-commodity flow, multiple services, capacitated multi-modal transportation and stochastic case comprehensively. This problem certainly matches the practice better than the current studies, but it also proposes great challenges for the researchers such as: (1) mathematical description of the transshipments among the multiple services; (2) formulation of the time related inventory capacity; and (3) stochastic analysis that needs massive reliable statistical data. Additionally, the solution algorithms for this problem also need to be developed.
Linearization technique, Lagrangian relaxation technique as well as the mentioned heuristic algorithms may be adopted or combined to solve this combinatorial optimization problem. How to test the effectiveness and efficiency of the solution algorithms is also a challenging work that we cannot avoid. In conclusion, freight routing planning problem in the multi-modal transportation network still has large research potential that needs to be explored.

Finally, we wish this review can draw more colleagues to the research of the freight routing planning problem in the multi-modal transportation network.

\section{CONFLICT OF INTEREST}

The authors confirm that this article content has no conflict of interest.

\section{ACKNOWLEDGEMENTS}

This study was supported by the National Natural Science Foundation Project (No.71390332-3) of People's Republic of China. The authors would thank the editor of the journal and the anonymous reviewers, for their constructive suggestions and comments that have led to a significant improvement of this paper.

\section{REFERENCES}

[1] A. Capone, G. Carello, I. Filippini, S. Gualandi, and F. Malucelli, "Routing, scheduling and channel assignment in wireless mesh networks: optimization models and algorithms", Ad Hoc Netw., vol. 8, pp. 545-563, August 2010.

[2] S. Ehsan, and B. Hamdaoui, "A survey on energy-efficient routing techniques with QoS assurances for wireless multimedia sensor networks”, IEEE Commun. Surv. Tutor., vol. 14, pp. 265-278, March 2012.

[3] M. Radi, B. Dezfouli, K. A. Bakar, and M. Lee, "Multipath routing in wireless sensor networks: survey and research challenges", Sensors, vol. 12, pp. 650-685, January 2012.

[4] O. Z. Maimon, and S. B. Gershwin, "Dynamic scheduling and routing for flexible manufacturing systems that have unreliable machines”, Oper. Res., vol. 36, pp. 279-292, April 1988.

[5] G. B. Gao, G. J. Zhang, G. Huang, H. P. Zhu, and P. H. Gu, "Solving material distribution routing problem in mixed manufacturing systems with a hybrid multi-objective evolutionary algorithm”, J. Cent. South Univ., vol. 19, pp. 433-442, January 2012.

[6] I. Eguia, S. Lozano, J. Racero, and F. Guerrero, “Cell design and loading with alternative routing in cellular reconfigurable manufacturing systems," In: $7^{\text {th }}$ IFAC Conference on Manufacturing Modelling, Management, and Control, Russia, 2013, pp. 1744-1749.

[7] B. Xiao, J. Cao, Z. Shao, and E. M. Sha, "An efficient algorithm for dynamic shortest path tree update in network routing", $J$. Commun. Netw., vol. 9, pp. 499-510, December 2007.

[8] L. Gao, and J. Rexford, "Stable Internet routing without global coordination”, IEEE ACM Trans. Netw., vol. 9, pp. 681-692, December 2001.

[9] Y. Lee, and B. Mukherjee, "A traffic engineering-aware shortestpath routing algorithm in IP networks", Lect. Notes Comput. Sci., vol. 3042, pp. 1204-1215, 2004.

[10] C. Macharis, and Y. M. Bontekoning, "Opportunities for OR in intermodal freight transport research: a review”, Eur. J. Oper. Res., vol. 153, pp. 400-416, March 2004.

[11] M. Yaghini, and R. Akhavan, "Multicommodity network design problem in rail freight transportation planning," In: $8^{\text {th }}$ International Conference on Traffic and Transportation Studies (ICTTS 2012), China, 2012, pp. 728-739. 
[12] T. S. Chang, "Best routes selection in international intermodal networks,” Comp. Oper. Res., vol. 35, pp. 2877-2891, September 2008.

[13] M. Janic, "Modelling the full costs of an intermodal and road freight transport network", Transp. Res. Part D Transp. Environ., vol. 12, pp. 33-44, January 2007.

[14] C. H. Liao, P. H. Tseng, and C. S. Lu, "Comparing carbon dioxide emissions of trucking and intermodal container transport in Taiwan”, Transp. Res. Part D Transp. Environ., vol. 14, pp. 493496, October 2009.

[15] L. Moccia, J. F. Cordeau, G. Laporte, S. Ropke, and M. P. Valentini, "Modeling and solving a multimodal transportation problem with flexible-time and scheduled services", Networks, vol. 57, pp. 53-68, April 2010.

[16] A. Caprara, E. Malaguti, and P. Toth, "A freight service design problem for a railway corridor”, Transp. Sci., vol. 45 pp. 147-162, May 2011.

[17] M. Boussedjra, C. Bloch, and A. El Moudni, "An exact method to find the intermodal shortest path (ISP)," IEEE Int. Conf. Netw. Sens. Control, vol. 2, pp. 1075-1080, 2004.

[18] G. W. Xiong, and Y. Wang, "Best routes selection in multimodal networks using multi-objective genetic algorithm”, J. Combin. Optim., vol. 28, pp. 655-673, October 2014.

[19] T. G. Crainic, M. Gendreau, and J. M. Farvolden, "A simplexbased tabu search method for capacitated network design", INFORMS J. Comput., vol. 12, pp. 223-236, August 2000.

[20] Y. M. Bontekoning, C. Macharis, and J. J. Trip, "Is a new applied transportation research field emerging?-A review of intermodal rail-truck freight transport literature", Transp. Res. Part A Policy Pract., vol. 38, pp. 1-34, January 2004.

[21] A. Caris, C. Macharis, and G. K. Janssens, "Planning problems in intermodal freight transport: accomplishments and prospects", Transp. Plan. Technol., vol. 31, pp. 277-302, June 2008.

[22] C. Barnhart, and H. D. Ratliff, "Modeling intermodal routing", $J$. Bus. Logist., vol. 14, pp. 205-223, 1993.

[23] K. Lei, X. Zhu, J. Hou, and W. Huang, "Decision of multimodal transportation scheme based on swarm intelligence", Math. Probl. Eng., vol. 2014, pp. 1-10, April 2014.

[24] T. G. Crainic, and J. M. Rousseau, "Multicommodity, multimode freight transportation: A general modeling and algorithmic framework for the service network design problem”, Transp. Res. Part B Methodol., vol. 20, pp. 225-242, June 1986.

[25] J. H. Bookbinder, and N. S. Fox, "Intermodal routing of CanadaMexico shipments under NAFTA", Transp. Res. Part E Logist. Transp. Rev., vol. 34, pp. 289-303, December 1998.

[26] J. Holguín-Veras, and G. A. Patil, "A multicommodity integrated freight origin-destination synthesis model”, Netw. Spat. Econ., vol. 8, pp. 309-326, January 2008.

[27] Y. H. Zhang, B. L. Lin, D. Liang, and H. Y. Gao, "Research on a generalized shortest path method of optimizing intermodal transportation problems", J. China Railw. Soc., vol. 28, pp. 22-26, August 2006.

[28] J. Liu, S. W. He, R. Song, and H. D. Li, "Study on optimization of dynamic paths of intermodal transportation network based on alternative set of transport modes”, J. China Railw. Soc., vol. 33, pp. 1-6, October 2011.

[29] Y. Li, J. Zhao, G. Wu, and J. Chen, "Solving the mode selection problem with fixed transportation cost in intermodal transportation”, J. Southwest Jiaotong Univ., vol. 47, pp. 881-887, October 2012.

[30] Y. Qu, T. Bektaş, and J. Bennell, "Sustainability SI: Multimode multicommodity network design model for intermodal freight transportation with transfer and emission Costs”, Netw. Spat. Econ., pp. 1-27, April 2014.

[31] A. Atamtürk, and D. Rajan, "On splittable and unsplittable flow capacitated network design arc-set polyhedra", Math. Program., vol. 92, pp. 315-333, April 2002.

[32] A. Balakrishnan, T. L. Magnanti, and R. T. Wong, "A dual-ascent procedure for large-scale uncapacitated network design”, Oper. Res., vol. 37, pp. 716-740, September - October 1989.

[33] Q. B. Wang, and Z. X. Han, "The optimal routes and modes selection in container multimodal transportation networks," Int. Conf. Optoelectron. Image Process., vol. 2, pp. 573-576, 2010.

[34] K. Holmberg, and D. Yuan, "A Lagrangian heuristic based branchand-bound approach for the capacitated network design problem”, Oper. Res., vol. 48, pp. 461-481, June 2000.
[35] J. H. Cho, H. S. Kim, and H. R. Choi, “An intermodal transport network planning algorithm using dynamic programming-a case study: from Busan to Rotterdam in intermodal freight routing”, Appl. Intell., vol. 36, pp. 529-541, April 2012.

[36] H. Sun, X. Li, and D. Chen, "Modeling and solution methods for viable routes in multimodal networks," In: $4^{\text {th }}$ IEEE International Conference on Management of Innovation and Technology, Thailand, 2008, pp. 1384-1388.

[37] J. F. Cordeau, G. Laporte, S. Ropke, and M. P. Valentini, Modeling and solving a multimodal routing problem with timetables and time windows, CIRRELT: Montréal, 2008.

[38] B. Ayar, and H. Yaman, "An intermodal multicommodity routing problem with scheduled services”, Comput. Optim. Appl., vol. 53, pp. 131-153, September 2012.

[39] H. Song, and G. Chen, "Minimum cost delivery problem in intermodal transportation networks," In: IEEE International Conference on Industrial Engineering and Engineering Management, Singapore, 2007, pp. 1502-1506.

[40] Y. Li, S. Tong, M. Li, and J. Zhao, "Solving mode selection problem in multimodal transportation considering transportation cost structure," In: $3^{\text {rd }}$ International Conference on Transportation Engineering (ICTE), China, 2011, p. 204-209.

[41] B. Sun, and Q. S. Chen, "The routing optimization for multi-modal transport with carbon emission consideration under uncertainty," In: $32^{\text {nd }}$ Chinese Control Conference, China, 2013, pp. 8135-8140.

[42] J. Andersson, A Survey of Multiobjective Optimization in Engineering Design, Department of Mechanical Engineering, Linktjping University, Sweden, 2000.

[43] A. Messac, A. Ismail-Yahaya, and C. A. Mattson, "The normalized normal constraint method for generating the Pareto frontier", Struct. Mutltidiscip. Opt., vol. 25, pp. 86-98, July 2003.

[44] M. Tamiz, D. Jones, and C. Romero, "Goal programming for decision making: An overview of the current state-of-the-art”, Eur. J. Oper. Res., vol. 111, pp. 569-581, December 1998.

[45] J. Jiang, and J. Lu, "Research on optimum combination of transportation modes in the container multimodal transportation system," In: $8^{\text {th }}$ International Conference of Chinese Logistics and Transportation Professionals (ICCLTP), China, pp. 693-698, 2009.

[46] R. A. Garrido, and H. S. Mahmassani, "Forecasting freight transportation demand with the space time multinomial probit model”, Transp. Res. Part B Methodol., vol. 34, pp. 403-418, June 2000.

[47] R. Bai, S. W. Wallace, J. Li, and A. Y. L. Chong, "Stochastic service network design with rerouting", Transp. Res. Part B Methodol., vol. 60, pp. 50-65, February 2014.

[48] Q. B. Wang, Z. X. Han, M. J. Ji, and Y. M. Li, 'Path optimization of container multimodal transportation based on node operation randomness", J. Transp. Syst. Eng. Inf. Technol., vol. 11, pp.137144, December 2011.

[49] T. Wang, and G. A. Wang, "Combined optimization model for transportation modes of multimodal transport”, Eng. Sci., vol. 7, pp. 46-50, October 2005.

[50] H. Wei, J. Li, and N. Z. Liu, "An algorithm for shortest path with multi-modal in time-varying network", Chin. J. Manage. Sci., vol. 14, pp. 56-58, August 2006.

[51] Y. P. Cai, L. Zhang, and L. N. Shao, "Optimal multi-modal transport model for full loads with time windows," In: International Conference on Logistics Systems and Intelligent Management, China, 2010, pp. 147-151.

[52] X. Wang, Z. B. Chi, and X. L. Ge, "Research and analysis for timelimited multimodal transport model of vehicle”, Appl. Res. Comput., vol. 28, pp. 563-565, June 2011.

[53] T. G. Crainic, Y. Li, and M. Toulouse, "A first multilevel cooperative algorithm for capacitated multicommodity network design”, Comp. Oper. Res., vol. 33, pp. 2602-2622, September 2006.

[54] M. X. Lang, and S. J. Hu, "Study of the optimizing of physical distribution routing problem based on genetic algorithm", China J. Highw. Transp., vol. 15, pp. 76-79, July 2002.

[55] M. X. Lang, and S. J. Hu, "Study on the optimization of physical distribution routing problem by using hybrid genetic algorithm", Chin. J. Manage. Sci., vol. 10, pp. 51-56, October 2002.

[56] M. Martínez, M., S. García-Nieto, J. Sanchis, and X. Blasco, "Genetic algorithms optimization for normalized normal constraint method under Pareto construction”, Adv. Eng. Software, vol. 40, pp. 260-267, April 2009. 
[57] D. W. Wang, J. W. Wang, R. Y. Zhang, and Z. Guo, Ed. Intelligent Optimization Methods. Higher Education Press, Beijing, 2007.

[58] Y. Sun, M. X. Lang, D. Z. Wang, and L. Y. Liu, "A PSO-GRNN model for railway freight volume prediction: Empirical study from China”, J. Ind. Eng. Manage., vol. 7, pp. 413-433, May 2014.
[59] F. Glover, J. P. Kelly, and M. Laguna, "Genetic algorithms and tabu search: hybrids for optimization”, Comp. Oper. Res., vol. 22, pp. 111-134, January 1995.

(C) Sun et al.; Licensee Bentham Open.

This is an open access article licensed under the terms of the Creative Commons Attribution Non-Commercial License (http://creativecommons.org/licenses/ by-nc/3.0/) which permits unrestricted, non-commercial use, distribution and reproduction in any medium, provided the work is properly cited. 